\title{
Características seminais de touros Curraleiros e Holandeses submetidos à insulação escrotal
}

\author{
Tomaz Gelson Pezzini(1), Roberto Sartori(2), Thiago Antônio de Souza Nascimento Silva ${ }^{(1)}$, \\ Concepta McManus ${ }^{(1)}$ e Arthur da Silva Mariante ${ }^{(1)}$
}

\begin{abstract}
(1)Universidade de Brasília, Fac. de Agronomia e Medicina Veterinária, Caixa Postal 4.508, CEP 70910-900 Brasília, DF. E-mail: tomaz@unb.br, thiagoasns@unb.br, concepta@unb.br (2)Embrapa Recursos Genéticos e Biotecnologia, Caixa Postal 02372, CEP 70770-900 Brasília, DF. E-mail: sartori@cenargen.embrapa.br, mariante@cenargen.embrapa.br
\end{abstract}

\begin{abstract}
Resumo - O objetivo deste trabalho foi comparar uma raça naturalizada brasileira com uma raça européia quanto à adaptação às altas temperaturas. Foram avaliados os efeitos da insulação escrotal, por 48 horas, nas características seminais de touros Curraleiros $(n=5)$ e Holandeses $(n=6)$. Uma sequiência de coletas de sêmen e avaliação andrológica dos touros foi realizada antes (duas coletas) e depois (nove coletas semanais) da insulação escrotal. A motilidade espermática progressiva teve queda significativamente menor na raça Curraleira (25\%) do que na Holandesa (50\%), no período pós-insulação, e retornou aos valores pré-insulação mais precocemente nos touros Curraleiros, aos 37 dias após a insulação escrotal. O vigor espermático nos Curraleiros $(15,2 \%)$ teve queda menor do que nos Holandeses (47,8\%). O número de espermatozóides por ejaculado caiu de forma similar nos dois grupos depois da insulação, respectivamente 67,9 e 79,4\%, para Curraleiros e Holandeses, entre 16 e 23 dias após a insulação. A porcentagem de espermatozóides anormais atingiu valores máximos entre 16 e 30 dias após a insulação e foi maior aos 30 dias nos touros Holandeses (41,3ะ5,9\%). Os touros Curraleiros são mais tolerantes aos efeitos da insulação escrotal nas características seminais do que os Holandeses.
\end{abstract}

Termos para indexação: reprodução animal, sêmen bovino, degeneração testicular, conservação de recursos genéticos.

\section{Seminal characteristics of Curraleiro and Holstein bulls submitted to scrotal insulation}

\begin{abstract}
The objective of this work was to compare the adaptation to high temperatures of a Brazilian local breed with an European one. The effects of scrotal insulation for 48 hours on seminal characteristics of Curraleiro $(n=5)$ versus Holstein $(n=6)$ bulls were evaluated. A sequence of semen collection and andrologic evaluation was performed before (two collections) and after (nine weekly collections) scrotal insulation. Among the seminal parameters evaluated, the progressive sperm motility had a significantly lower decrease in Curraleiro (25\%) than in Holstein bulls (50\%), during the postinsulation period. Sperm motility of Curraleiro bulls, 37 days after insulation, returned to pre-insulation values sooner than that of Holstein bulls (51 days). Sperm vigor had a lower decrease after insulation in Curraleiro (15.2\%) than in Holstein bulls (47.8\%). The total number of spermatozoa per ejaculate has dropped similarly in both groups after scrotal insulation (67.9 and $79.4 \%$ of drop for Curraleiro and Holstein bulls, respectively, between 16 and 23 days). The percentage of abnormal spermatozoa reached maximum values between 16 and 30 days after insulation and was greater in Holstein bulls (41.3 $\pm 5.9 \%$ ) 30 days after the insult. Curraleiro are more tolerant to the effects of scrotal insulation on seminal characteristics than Holstein bulls.
\end{abstract}

Index terms: animal reproduction, bovine semen, testicular degeneration, genetic resources conservation.

\section{Introdução}

O efeito da temperatura sobre a qualidade seminal já foi estudado em diversas espécies domésticas. Em bovinos, a degeneração testicular por efeito térmico é a principal causa de infertilidade e sub-fertilidade (Garcia, 1971; McEntee, 1973).
Em condições idênticas de criação, freqüentemente são detectadas diferenças no desempenho reprodutivo entre raças brasileiras naturalizadas e raças exóticas taurinas, considerando-se a diversidade genética entre as duas subespécies. Sob condições de clima tropical, observou-se que a elevada temperatura e umidade do ar podem deprimir as funções gonadais e aumentar o 
número de touros com degeneração testicular (Horn et al., 1997).

Galina \& Arthur (1991), em uma revisão sobre aspectos reprodutivos em touros nos trópicos, salientam a importância da variação sazonal sobre a concentração espermática e a porcentagem de espermatozóides com defeitos morfológicos, indicando que a baixa qualidade do sêmen, em alguns animais, pode ocorrer em razão do desconforto causado por temperaturas ambientais elevadas. Fonseca et al. (1992), ao estudar a raça Nelore, também concluíram que a qualidade do sêmen pode ser afetada significativamente pelo ambiente, por meio de flutuações na temperatura, umidade e fotoperíodo.

É sabido que existem diferenças entre o comportamento reprodutivo das raças naturalizadas brasileiras, quando comparadas a raças comerciais (Santos \& Simplício, 2000). Entre as formas de se avaliar a eficiência reprodutiva de uma raça em determinado ambiente específico, a insulação escrotal por curtos períodos mostra-se como uma opção na avaliação reprodutiva de touros em climas quentes.

O objetivo deste trabalho foi avaliar os efeitos da insulação escrotal nas características seminais e morfométricas testiculares da raça Curraleira, uma das raças naturalizadas brasileiras, e comparar aos mesmos parâmetros na raça Holandesa.

\section{Material e Métodos}

O experimento foi realizado no período de outubro a dezembro de 2004, no Campo Experimental Sucupira, pertencente à Embrapa Recursos Genéticos e Biotecnologia, em Brasília, DF. Foram utilizados seis touros da raça Curraleira (Bos taurus ibericus) e seis touros da raça Holandesa (Bos taurus taurus). Os animais encontravam-se clinicamente saudáveis, sexualmente maduros, com idade média de seis anos, com variação de cinco a oito anos nos touros Curraleiros e de quatro a sete anos nos Holandeses.

Os touros apresentavam histórico de boa fertilidade e estavam condicionados à coleta de sêmen por eletroejaculação. Durante o início da fase experimental, um dos touros Curraleiros foi removido do experimento por ter sofrido uma lesão no membro anterior esquerdo. Seus dados, portanto, não foram utilizados. Os animais foram mantidos em pastagem cultivada de Brachiaria decumbens, com suplementação mineral e água ad libitum, durante todo o período experimental.
O experimento compreendeu três fases. A fase de pré-insulação escrotal correspondeu à primeira semana do experimento, em que se procedeu à avaliação andrológica completa dos touros, com coleta e avaliação de sêmen nas duas raças estudadas. A fase de insulação escrotal correspondeu ao período em que os animais permaneceram com uma bolsa térmica nos testículos, pelo período de 48 horas, a fim de simular a interferência ambiental na termorregulação testicular de touros em climas quentes (Vogler et al., 1991). A fase de pós-insulação correspondeu ao período após a retirada da bolsa térmica dos animais, em que se procedeu novamente à avaliação andrológica completa nos touros, com coleta e avaliação de sêmen nas duas raças. Os valores obtidos no período pós-insulação foram comparados à média dos valores pré-insulação.

Os animais foram divididos em dois grupos, conforme a raça. Cada animal foi avaliado por exame andrológico completo. Na primeira fase experimental, foram feitas três coletas de sêmen em cada animal, antes da insulação escrotal, com intervalos de três dias entre coletas. A primeira coleta de sêmen foi realizada para remover os espermatozóides envelhecidos e degenerados da cauda do epidídimo dos animais. Nas duas coletas subseqüentes, foram mensurados os parâmetros morfométricos testiculares e características seminais. Imediatamente depois da última coleta, foi feita a insulação escrotal em todos os animais com uma bolsa térmica (fralda descartável), constituída por uma dupla camada plástica intermediada por uma camada de algodão, similar à usada no estudo de Brito et al. (2003). Essa bolsa foi fixada com esparadrapo ao escroto, de forma que os touros não pudessem removê-la, entretanto, manteve-se o cuidado para que a bolsa não provocasse interferência na circulação escrotal. Depois da retirada da bolsa, o sêmen foi coletado, em intervalos semanais, até que se completasse um período de 58 dias. Uma coleta adicional foi realizada três dias depois da retirada da bolsa térmica, para se avaliar o efeito imediato da insulação escrotal nos parâmetros seminais.

O sêmen foi coletado por meio de eletroejaculação e avaliado de acordo com as normas do Colégio Brasileiro de Reprodução Animal (1998), quanto aos seguintes parâmetros: volume, vigor, motilidade progressiva, concentração espermática, número total de espermatozóides do ejaculado e porcentagem de espermatozóides anormais. As alterações morfológicas espermáticas foram classificadas de acordo com Barth \& Oko (1989). 
Os parâmetros analisados no período pré-insulação foram relacionados com as duas raças estudadas e com os diferentes dias de coleta do período pós-insulação escrotal. Os parâmetros de motilidade espermática progressiva, vigor espermático, número total de espermatozóides do ejaculado, porcentagem de espermatozóides anormais, gota citoplasmática proximal, cauda fortemente dobrada ou enrolada, cabeça isolada normal e cauda dobrada ou enrolada foram analisados segundo um modelo com as seguintes fontes de variação: raça (Curraleira e Holandesa), tratamento (período pré-insulação e pós-insulação escrotal) e período pósinsulação escrotal em 5, 9, 16, 23, 30, 37, 44, 51 e 58 dias. Estas avaliações foram consideradas como medidas repetidas, observadas na mesma unidade experimental e incorporadas ao modelo como um vetor de variável resposta.

Procedeu-se à análise de variância segundo o modelo inicialmente proposto para cada período de coleta de dados, sendo, para tal, utilizado o procedimento GLM, do programa Statistical Analysis System (SAS). As múltiplas comparações e intervalos de confiança foram obtidos pelo teste t. No caso dos parâmetros com medidas repetidas, utilizou-se, inicialmente, a opção "Repeated" para realizar os testes das hipóteses de paralelismo e decomposição dos efeitos, no decorrer do período experimental.

\section{Resultados e Discussão}

A insulação escrotal causou uma redução significativa na motilidade espermática, a partir do quinto e nono dias após a aplicação da bolsa térmica nos touros Holandeses e Curraleiros, respectivamente (Tabela 1). Estes resultados divergem dos encontrados em outros trabalhos, que relataram a ocorrência de alteração na motilidade espermática apenas a partir do $12^{\circ}$ dia, após a insulação escrotal (Ross \& Entwistle, 1979; Wildeus \& Entwistle, 1983; Vogler et al., 1993; Barth \& Bowman, 1994; Brito et al., 2003). Neste trabalho, supõe-se que o efeito do aumento da temperatura, na bolsa escrotal, tenha se evidenciado primeiramente no epidídimo, interferindo no processo de maturação da célula espermática.

Verificou-se, ainda, que houve diferença significativa entre raças, no declínio inicial da motilidade progressiva, e no tempo de retorno dessa aos valores pré-insulação (Tabela 1). Há evidências de que, nos touros Holandeses, as espermátides foram afetadas também durante a espermatocitogênese, enquanto na raça Curraleira, aparentemente, somente as espermátides em fase de maturação foram afetadas pela insulação escrotal. Tais resultados podem ser traduzidos como maior ou menor adaptação dos animais ao meio tropical e são semelhantes aos descritos por Troncoso (1997), quando avaliou a qualidade de sêmen de touros Simental, em relação à tolerância ao calor.

As células germinativas testiculares apresentam diferentes graus de sensibilidade à elevação de temperatura escrotal. Espermatócitos e espermátides são particularmente sensíveis, enquanto que as espermatogônias são mais resistentes (Setchell, 1998). Após a insulação escrotal, mudanças na produção e qualidade espermática ocorrem depois de um intervalo determinado, que varia de acordo com o estádio de desenvolvimento das células germinativas afetadas no momento da insulação escrotal (Barth \& Oko, 1989).

O vigor espermático e o número total de espermatozóides do ejaculado responderam de forma diferenciada à insulação escrotal, quando as duas raças estudadas foram comparadas (Tabela 1). O vigor espermático apresentou uma diminuição significativa nos touros da raça Holandesa, chegando a apresentar 50\% dos valores pré-insulação, aos 16 dias depois da insulação escrotal. Nesses animais, houve, também, um comprometimento direto ou mesmo indireto da célula espermática, ao longo do processo de maturação no epidídimo. Os touros da raça Curraleira apresentaram apenas uma discreta variação no vigor espermático, durante todo o período experimental, não tendo havido influência da insulação escrotal em relação ao epidídimo, para essa raça. Em relação ao número total de espermatozóides do ejaculado, observa-se nas duas raças avaliadas uma diminuição nesses valores, a partir de cinco a nove dias até 37 a 44 dias após a insulação escrotal (Tabela 1). No entanto, entre os nove e 16 dias do período pós-insulação, o número total de espermatozóides nos touros da raça Curraleira representa duas vezes os valores apresentados pelos animais da raça Holandesa.

Variações significativas também foram observadas na avaliação da morfologia espermática, em todos os parâmetros mensurados. Houve diferença na porcentagem de espermatozóides anormais, quando foram comparadas as duas raças estudadas. Touros da raça Curraleira apresentaram, ao redor do 16ํ dia após a insulação escrotal, valores máximos de espermatozóides anormais no ejaculado. A raça Holandesa, no entanto, 
manteve-se acima de $30 \%$ de anormalidades espermáticas, entre o 16o e 440 dia após a insulação (Tabela 1).

Em estudos anteriores com animais taurinos, a insulação escrotal também resultou no aumento de espermatozóides anormais, com retorno aos parâmetros normais entre o 32 e o 42 dia após a insulação escrotal (Austin et al., 1961; Vogler et al., 1993; Barth \& Bowman, 1994). Em outros experimentos, verificou-se que a insulação escrotal em zebuínos, por períodos mais longos, de 96 e 168 horas, resultaram em maior e mais intensa redução na motilidade e morfologia espermática, e os animais necessitaram de 105 e 148 dias, respectivamente, para retornarem aos valores de normalidade espermática (Fonseca \& Chow, 1995; Gabaldi et al., 1999).

O mecanismo pelo qual a insulação escrotal leva à formação de espermatozóides anormais é discutido por diversos autores. Setchell (1998) sugere que o aumento de temperatura testicular resulta num incremento do metabolismo e demanda de oxigênio, de tal forma que a vascularização sangüínea testicular não consegue neutralizá-la, levando à hipóxia e formação de um oxigênio reativo e, conseqüentemente, à formação de anormalidades espermáticas.

Barth \& Oko (1989) observaram que existe uma predisposição genética para o desenvolvimento de alterações morfológicas espermáticas específicas, em resposta a condições adversas como o aumento da temperatura testicular. Esta afirmação está de acordo com os resul- tados obtidos neste experimento, visto que apenas quatro tipos de alterações morfológicas espermáticas - gota citoplasmática proximal, cauda fortemente dobrada ou enrolada, cabeça isolada normal e cauda dobrada ou enrolada - diferiram significativamente após a insulação escrotal nas duas raças.

Todos os espermatozóides que chegam ao epidídimo apresentam gota citoplasmática na posição proximal. A migração da gota citoplasmática da posição proximal para distal ocorre durante a maturação espermática, ao longo da passagem do espermatozóide pelo epidídimo. A gota distal é perdida no momento da ejaculação, por meio dos movimentos de cauda espermática (Barth \& Oko, 1989; Amann, 1991).

Gabaldi et al. (1999) relatou um aumento de gota proximal entre o sétimo e o décimo dia do período pósinsulação, sugerindo um comprometimento da função do epidídimo no aparecimento desta alteração morfológica. No entanto, no presente experimento, observou-se que o aumento significativo de gota citoplasmática proximal ocorreu apenas a partir do $23^{\circ}$ dia, o que indica que a ocorrência de gota citoplasmática proximal no ejaculado foi resultado da alteração de espermátides ou mesmo de espermatócitos. Verificou-se, ainda, que existe diferença significativa entre raças, com valores de picos de gota citoplasmática proximal que variam de 3,5\% na raça Curraleira, a 13\% na Holandesa (Tabela 2).

Observou-se um aumento de cabeça isolada normal, a partir do quinto dia após a insulação (Tabela 2), com

Tabela 1. Valores médios ( \pm erro-padrão) de motilidade espermática progressiva (\%), vigor espermático, número total de espermatozóides do ejaculado e espermatozóides anormais $(\%)$, de touros Holandeses $(n=6)$ e Curraleiros $(n=5)$ submetidos à insulação escrotal por 48 horas, ao longo de 58 dias $^{(1)}$.

\begin{tabular}{|c|c|c|c|c|c|c|c|c|}
\hline \multirow{2}{*}{$\begin{array}{c}\text { Dias após } \\
\text { insulação escrotal }\end{array}$} & \multicolumn{2}{|c|}{ Motilidade espermática } & \multicolumn{2}{|c|}{ Vigor espermático } & \multicolumn{2}{|c|}{ Espermatozóides totais } & \multicolumn{2}{|c|}{ Espermatozóides anormais } \\
\hline & Holandês & Curraleiro & Holandês & Curraleiro & Holandês & Curraleiro & Holandês & Curraleiro \\
\hline 0 & $73,0 \pm 1,7 \mathrm{aA}$ & $64,0 \pm 2,5 \mathrm{bA}$ & $3,8 \pm 0,1 \mathrm{aA}$ & $3,2 \pm 0,1 \mathrm{bA}$ & $2.343,0 \pm 466,2 \mathrm{~A}$ & $2.505,0 \pm 604,0 \mathrm{~A}$ & $14,5 \pm 2,5 \mathrm{~B}$ & $8,2 \pm 0,8 \mathrm{~B}$ \\
\hline 5 & $43,3 \pm 3,3 \mathrm{bB}$ & $56,0 \pm 2,4 \mathrm{aA}$ & $3,0 \pm 0,3 \mathrm{~B}$ & $3,2 \pm 0,2 \mathrm{~A}$ & $1.016,7 \pm 178,0 \mathrm{~B}$ & $1.708,0 \pm 613,7 \mathrm{~A}$ & $23,7 \pm 2,7 \mathrm{~A}$ & $24,0 \pm 7,0 \mathrm{~B}$ \\
\hline 9 & $38,3 \pm 3,1 \mathrm{bB}$ & $48,0 \pm 2,0 \mathrm{aB}$ & $2,7 \pm 0,2 \mathrm{~B}$ & $2,8 \pm 0,2 \mathrm{~A}$ & $510,0 \pm 63,1 \mathrm{~B}$ & $1.014,0 \pm 346,0 \mathrm{~B}$ & $29,0 \pm 3,1 \mathrm{~A}$ & $25,4 \pm 3,7 \mathrm{~A}$ \\
\hline 16 & $36,7 \pm 2,1 \mathrm{bB}$ & $48,0 \pm 2,0 \mathrm{aB}$ & $2,0 \pm 0,0 \mathrm{bB}$ & $2,8 \pm 0,2 \mathrm{aB}$ & $483,3 \pm 74,2 \mathrm{bB}$ & $974,0 \pm 182,0 \mathrm{aB}$ & $38,7 \pm 6,9 \mathrm{~A}$ & $32,0 \pm 2,6 \mathrm{~A}$ \\
\hline 23 & $38,3 \pm 1,7 \mathrm{bB}$ & $50,0 \pm 0,0 \mathrm{aB}$ & $2,3 \pm 0,2 \mathrm{bB}$ & $3,0 \pm 0,0 \mathrm{aA}$ & $705,0 \pm 150,5 B$ & $804,0 \pm 200,2 \mathrm{~B}$ & $34,5 \pm 4,3 \mathrm{~A}$ & $24,8 \pm 2,1 \mathrm{~A}$ \\
\hline 30 & $41,7 \pm 1,7 \mathrm{bB}$ & $54,0 \pm 2,4 \mathrm{aB}$ & $2,5 \pm 0,2 \mathrm{~B}$ & $3,0 \pm 0,0 \mathrm{~A}$ & $835,0 \pm 162,2 \mathrm{~B}$ & $1.264,0 \pm 517,4 \mathrm{~B}$ & $41,3 \pm 5,9 \mathrm{aA}$ & $21,8 \pm 7,6 \mathrm{bA}$ \\
\hline 37 & $50,0 \pm 2,6 \mathrm{bB}$ & $64,0 \pm 2,4 \mathrm{aA}$ & $2,5 \pm 0,2 \mathrm{~B}$ & $3,2 \pm 0,4 \mathrm{~A}$ & $1.180,0 \pm 274,3 \mathrm{~A}$ & $962,0 \pm 173,3 \mathrm{~B}$ & $38,2 \pm 4,9 \mathrm{aA}$ & $23,2 \pm 2,3 \mathrm{bA}$ \\
\hline 44 & $55,0 \pm 2,2 \mathrm{bB}$ & $62,0 \pm 2,0 \mathrm{aA}$ & $2,8 \pm 0,2 \mathrm{~B}$ & $3,2 \pm 0,2 \mathrm{~A}$ & $1.986,7 \pm 527,7 \mathrm{~A}$ & $1.543,0 \pm 432,6 \mathrm{~A}$ & $31,2 \pm 5,3 \mathrm{aA}$ & $15,2 \pm 1,7 \mathrm{bA}$ \\
\hline 51 & $68,3 \pm 3,0 \mathrm{~A}$ & $76,0 \pm 2,4 \mathrm{~B}$ & $3,3 \pm 0,3 \mathrm{~A}$ & $3,6 \pm 0,2 \mathrm{~A}$ & $2.058,3 \pm 142,3 \mathrm{~A}$ & $2.238,0 \pm 752,6 \mathrm{~A}$ & $25,8 \pm 6,1 \mathrm{aB}$ & $9,8 \pm 1,1 \mathrm{bB}$ \\
\hline 58 & $65,0 \pm 3,4 \mathrm{~A}$ & $68,0 \pm 2,4 \mathrm{~A}$ & $3,0 \pm 0,3 \mathrm{~B}$ & $2,8 \pm 0,2 \mathrm{~B}$ & $2.350,0 \pm 756,6 \mathrm{~A}$ & $1.966,0 \pm 454,8 \mathrm{~A}$ & $17,0 \pm 4,4 \mathrm{~B}$ & $7,0 \pm 0,5 \mathrm{~B}$ \\
\hline Efeito de raça & $\mathrm{p}=0,001$ & & $\mathrm{p}=0,026$ & & $\mathrm{p}=0,645$ & & $\mathrm{p}=0,038$ & \\
\hline Efeito de dia & $\mathrm{p}<0,001$ & & $\mathrm{p}<0,001$ & & $\mathrm{p}<0,001$ & & $\mathrm{p}<0,001$ & \\
\hline Interação raça $\mathrm{x}$ dia & $\mathrm{p}<0,001$ & & $\mathrm{p}=0,053$ & & $\mathrm{p}=0,801$ & & $\mathrm{p}=0,059$ & \\
\hline
\end{tabular}


Tabela 2. Valores porcentuais médios ( \pm erro-padrão) de gota citoplasmática proximal, cabeça isolada normal, cauda fortemente dobrada ou enrolada e cauda dobrada ou enrolada, de touros Holandeses $(n=6)$ e Curraleiros $(n=5)$ submetidos à insulação escrotal, por 48 horas, ao longo dos 58 dias do período experimental ${ }^{(1)}$.

\begin{tabular}{|c|c|c|c|c|c|c|c|c|}
\hline \multirow{2}{*}{$\begin{array}{l}\text { Dias após } \\
\text { insulação } \\
\text { escrotal }\end{array}$} & \multicolumn{2}{|c|}{ Gota citoplasmática proximal } & \multicolumn{2}{|c|}{ Cabeça isolada normal } & \multicolumn{2}{|c|}{$\begin{array}{c}\text { Cauda fortemente dobrada } \\
\text { ou enrolada }\end{array}$} & \multicolumn{2}{|c|}{ Cauda dobrada ou enrolada } \\
\hline & Holandês & Curraleiro & Holandês & Curraleiro & Holandês & Curraleiro & Holandês & Curraleiro \\
\hline 0 & $1,2 \pm 0,6 \mathrm{~B}$ & $0,8 \pm 0,2 \mathrm{~B}$ & $0,5 \pm 0,3 \mathrm{~B}$ & $0,3 \pm 0,2 \mathrm{~B}$ & $6,9 \pm 2,0 \mathrm{~B}$ & $3,9 \pm 0,5 \mathrm{~B}$ & $0,3 \pm 0,2 \mathrm{~B}$ & $0,2 \pm 0,3 \mathrm{~B}$ \\
\hline 5 & $0,3 \pm 0,2 \mathrm{~B}$ & $0,0 \pm 0,0 \mathrm{~B}$ & $0,7 \pm 0,6 \mathrm{~B}$ & $0,8 \pm 0,6 \mathrm{~B}$ & $6,0 \pm 1,2 \mathrm{~B}$ & $7,4 \pm 4,7 \mathrm{~B}$ & $15,2 \pm 1,5 \mathrm{~A}$ & $13,4 \pm 8,8 \mathrm{~A}$ \\
\hline 9 & $0,5 \pm 0,3 \mathrm{~B}$ & $0,6 \pm 0,4 \mathrm{~B}$ & $8,3 \pm 2,9 \mathrm{~A}$ & $3,2 \pm 2,5 \mathrm{~A}$ & $8,7 \pm 0,9 \mathrm{~B}$ & $5,8 \pm 2,6 \mathrm{~B}$ & $9,3 \pm 2,0 \mathrm{~A}$ & $14,6 \pm 6,3 \mathrm{~A}$ \\
\hline 16 & $1,7 \pm 1,3 \mathrm{~B}$ & $0,8 \pm 0,6 \mathrm{~B}$ & $5,3 \pm 3,6 \mathrm{~A}$ & $5,8 \pm 2,8 \mathrm{~A}$ & $14,3 \pm 2,1 \mathrm{~A}$ & $15,2 \pm 3,7 \mathrm{~A}$ & $11,3 \pm 4,2 \mathrm{~A}$ & $7,0 \pm 2,9 \mathrm{~A}$ \\
\hline 23 & $10,0 \pm 1,6 \mathrm{Aa}$ & $3,6 \pm 0,4 \mathrm{Ab}$ & $11,0 \pm 3,5 \mathrm{~A}$ & $9,4 \pm 1,8 \mathrm{~A}$ & $9,2 \pm 1,6 \mathrm{~B}$ & $1,2 \pm 2,1 \mathrm{~B}$ & $0,0 \pm 0,0 \mathrm{~B}$ & $0,0 \pm 0,0 \mathrm{~B}$ \\
\hline 30 & $13,0 \pm 2,5 \mathrm{Aa}$ & $4,5 \pm 0,8 \mathrm{Ab}$ & $12,7 \pm 3,9 \mathrm{~A}$ & $7,8 \pm 1,4 \mathrm{~A}$ & $7,5 \pm 1,2 \mathrm{~B}$ & $5,2 \pm 2,0 \mathrm{~B}$ & $0,0 \pm 0,0 \mathrm{~B}$ & $0,0 \pm 0,0 \mathrm{~B}$ \\
\hline 37 & $13,0 \pm 3,1 \mathrm{Aa}$ & $5,0 \pm 0,6 \mathrm{Ab}$ & $10,5 \pm 1,6 \mathrm{~A}$ & $7,2 \pm 1,0 \mathrm{~A}$ & $6,5 \pm 1,0 \mathrm{~B}$ & $4,8 \pm 1,4 \mathrm{~B}$ & $0,0 \pm 0,0 \mathrm{~B}$ & $0,0 \pm 0,0 \mathrm{~B}$ \\
\hline 44 & $11,3 \pm 2,5 \mathrm{Aa}$ & $4,0 \pm 0,5 \mathrm{Ab}$ & $9,7 \pm 2,9 \mathrm{~A}$ & $4,0 \pm 0,7 \mathrm{~A}$ & $5,5 \pm 1,0 \mathrm{~B}$ & $4,4 \pm 1,5 \mathrm{~B}$ & $0,0 \pm 0,0 \mathrm{~B}$ & $0,0 \pm 0,0 \mathrm{~B}$ \\
\hline 51 & $8,2 \pm 2,6 \mathrm{Aa}$ & $1,8 \pm 0,2 \mathrm{Ab}$ & $6,2 \pm 2,6 \mathrm{~B}$ & $2,0 \pm 0,3 \mathrm{~A}$ & $4,2 \pm 1,0 \mathrm{~B}$ & $2,4 \pm 0,7 \mathrm{~B}$ & $0,0 \pm 0,0 \mathrm{~B}$ & $0,0 \pm 0,0 \mathrm{~B}$ \\
\hline 58 & $4,8 \pm 1,9 \mathrm{~B}$ & $0,8 \pm 0,4 \mathrm{~B}$ & $4,2 \pm 2,2 \mathrm{~B}$ & $1,2 \pm 0,4 \mathrm{~A}$ & $5,2 \pm 0,6 \mathrm{~B}$ & $3,0 \pm 1,0 \mathrm{~B}$ & $0,0 \pm 0,0 \mathrm{~B}$ & $0,4 \pm 0,9 \mathrm{~B}$ \\
\hline Efeito de raça & $\mathrm{p}=0,038$ & & $\mathrm{p}=0,293$ & & $\mathrm{p}=0,411$ & & $\mathrm{p}=0,945$ & \\
\hline Efeito de dia & $\mathrm{p}<0,001$ & & $\mathrm{p}<0,001$ & & $\mathrm{p}<0,001$ & & $\mathrm{p}<0,001$ & \\
\hline Interação raça $x$ dia & $\mathrm{p}<0,001$ & & $p=0,423$ & & $p=0,914$ & & $\mathrm{p}=0,343$ & \\
\hline
\end{tabular}

(1)Médias seguidas de letras iguais, minúsculas e maiúsculas nas linhas e colunas, respectivamente, não diferem entre si a 5\% de probabilidade pelo teste $\mathrm{t}$.

pico de incidência no 23o dia para a raça Curraleira (9\%), e no 30 o dia para a Holandesa (13\%). O aumento no aparecimento de cabeças isoladas normais a partir do quinto dia indica que a alteração da função do epidídimo, pela insulação escrotal, representa um papel importante na patogenia desta alteração - coerente com os relatos de outros autores (Wildeus \& Entwistle, 1983; Barth \& Bowman, 1994; Gabaldi et al., 1999). Entretanto, a continuidade de aparecimento e elevação dessa alteração morfológica espermática, no decorrer do período de coletas, sugere que a conexão da cabeça com a peça intermediária do espermatozóide não se estabelece por completo, em razão de falhas na formação da lâmina basal que recobre esta ligação. Essa falha de formação da lâmina basal poderia ser resultante da alteração de espermátides, pela insulação escrotal, durante a fase de espermiogênese (Barth \& Oko, 1989).

Peças intermediárias dobradas e enroladas são defeitos adquiridos, provavelmente, ao longo do epidídimo, em consequiência da exposição da célula espermática às secreções epididimárias anormais (Barth \& Oko, 1989; Barth, 1993). A cauda do epidídimo tem propriedades que permitem aos espermatozóides serem armazenados por várias semanas, mantendo suas características de motilidade e fertilidade, adquiridas ao longo de sua passagem pelo epidídimo (Barth \& Oko, 1989; Amann, 1991). No entanto, para exercer a função de armazenar espermatozóides viáveis, a cauda do epidídimo tem que estar a uma temperatura constante- mente mais baixa que a corporal ou, até mesmo, que a temperatura testicular.

A incidência de cauda fortemente dobrada (Tabela 2) diferiu do período pré-insulação apenas no $16^{\circ}$ dia, não tendo apresentado diferença significativa entre as duas raças estudadas. Em relação à cauda dobrada ou enrolada (Tabela 2), verificou-se que sua incidência ocorreu do quinto ao 160 dia após a insulação escrotal, não tendo havido diferença significativa entre raças. Esta observação de cauda dobrada ou enrolada ocorreu no período em que as células afetadas encontravam-se no epidídimo, o que concorda com o relatado por outros autores (Swanson \& Boyd, 1962; Barth \& Oko, 1989; Barth, 1993).

\section{Conclusões}

1. Os touros Curraleiros mostram-se mais tolerantes aos efeitos da insulação escrotal nas características seminais do que os Holandeses.

2. Em condições ambientais desfavoráveis, como altas temperaturas e baixa oferta de alimentos, o uso de raças bovinas adaptadas é uma alternativa promissora.

\section{Referências}

AMANN, R.P. Anatomy and physiology of the testis and epididymis. In: SOCIETY FOR THERIOGENOLOGY ANNUAL MEETING, 1991, San Diego. Proceedings. San Diego, 1991. p.1-7. 
AUSTIN, J.W.; HUPP, E.W.; MURPHEE, R.L. Effects of scrotal insulation on semen of Hereford bulls. Journal of Animal Science, v.20, p.307-310, 1961.

BARTH, A.D. Insights to the pathogenesis of sperm abnormalities in bulls. In: CONGRESSO BRASILEIRO DE REPRODUÇÃO ANIMAL, 10., 1993, Belo Horizonte. Anais. Belo Horizonte: Colégio Brasileiro de Reprodução Animal, 1993. p.1-14.

BARTH, A.D.; OKO, R.J. Abnormal morphology of bovine spermatozoa. $1^{\text {st }}$ ed. Ames: Iowa State University Press, 1989. 285p.

BARTH, A.D.; BOWMAN, P.A. The sequential appearance of sperm abnormalities after scrotal insulation or dexamethasone treatment in bulls. Canadian Veterinary Journal, v.35, p.93-102, 1994.

BRITO, L.F.C.; SILVA, A.E.D.F.; BARBOSA, R.T.; UNANIAN, M.M.; KASTELIC, J.P. Effects of scrotal insulation on sperm production, semen quality, and testicular echotexture in Bos indicus and Bos indicus x Bos taurus bulls. Animal Reproduction Science, v.79, p.1-15, 2003.

COLÉGIO BRASILEIRO DE REPRODUÇÃO ANIMAL. Manual para exame andrológico e avaliação de sêmen animal. 2.ed. Belo Horizonte, 1998. 49p.

FONSECA, V.O.; CHOW, L.A. Seminal characteristics of zebu bulls with transitory testicular degeneration. Arquivo Brasileiro de Medicina Veterinária e Zootecnia, v.47, p.707-716, 1995.

FONSECA, V.O.; CRUDELI, G.A.; COSTA E SILVA, E.V.; HERMANNY, A. Aptidão reprodutiva de touros da raça Nelore: efeito de diferentes estações do ano sobre as características seminais, circunferência escrotal e fertilidade. Arquivo Brasileiro de Medicina Veterinária e Zootecnia, v.44, p.7-15, 1992.

GABALDI, S.H.; DEFINE, R.M.; BARROS, C.M.Q.; MASCARO, K.; KASTELIC, J.P.; ROSA, G.J.M. Efeitos da elevação da temperatura testicular nas características espermáticas em touros Nelore. Revista Brasileira de Reprodução Animal, v.23, p.222224, 1999.

GALINA, C.S.; ARTHUR, G.H. Review of cattle reproduction in the tropics. Part 6. The Male. Animal Breeding Abstracts, v.59, p.403-412, 1991.
GARCIA, O.S. Características físicas e morfológicas do sêmen de touros normais e de touros com distúrbios reprodutivos, de raças européias e indianas, criadas no Estado de Minas Gerais. 1971. 61p. Dissertação (Mestrado) - Universidade Federal de Minas Gerais, Belo Horizonte.

HORN, M.M.; MORAES, J.C.F.; GALINA, C.S. Qualidade de sêmen de touros Aberdeen Angus e Ibagé frente à degeneração testicular experimental. Archivo Latinoamericano de Producción Animal, v.5, p.356-359, 1997.

McENTEE, K. Reproductive pathology. New York: State Veterinary College, 1973. 159p.

ROSS, A.D.; ENTWISTLE, K.W. The effect of scrotal insulation on spermatozoal morphology and the rates of spermatogenesis and epididymal passage of spermatozoa in the bull. Theriogenology, v.11, p.111-129, 1979.

SANTOS, D.O.; SIMPLÍCIO, A.A. Parâmetros escroto-testiculares e de sêmen em caprinos adultos submetidos à insulação escrotal. Pesquisa Agropecuária Brasileira, v.35, p.1835-1841, 2000.

SETCHELL, B.P. The parkes lecture. Heat and the testis. Journal of Reproduction and Fertility, v.114, p.179-194, 1998.

SWANSON, E.W.; BOYD, L.J. Factors affecting coiled-tail spermatozoa in the bull. American Journal of Veterinary Research, v.23, p.300-309, 1962.

TRONCOSO, E.U. Tolerância ao calor em touros da raça Simental e sua relação com a qualidade de sêmen. 1997. 82p. Dissertação (Mestrado) - Universidade de Brasília, Brasília.

VOGLER, C.J.; BAME, J.H.; DeJARNETTE, J.M.; McGILLIARD, M.L.; SAACKE, R.G. Effects of elevated testicular temperature on morphology characteristics of ejaculated spermatozoa in the bovine. Theriogenology, v.40, p.1207-1219, 1993.

VOGLER, C.J.; SAACKE, R.G.; BAME, J.H.; DeJARNETTE, J.M.; McGILLIARD, M.L. Effects of scrotal insulation on viability characteristics of cryopreserved bovine semen. Journal of Dairy Science, v.74, p.3827-3835, 1991.

WILDEUS, S.; ENTWISTLE, K.W. Spermiogram and sperm reserves in hybrid Bos indicus x Bos taurus bulls after scrotal insulation. Journal of Reproduction and Fertility, v.69, p.711-716, 1983.

Recebido em 29 de março de 2005 e aprovado em 7 de dezembro de 2005 